\title{
Metodología para Seleccionar Aplicaciones de la Tecnología de Comunicaciones Voz sobre la IP (VoIP) para Pequeñas y Medianas Empresas (Pymes). El caso de una Agencia de Viajes
}

\author{
Raúl J. Martelo ${ }^{(1)}$ Isamar Blanquicet y Lilibeth Rodríguez \\ (1) Universidad de Cartagena, Facultad de Ingeniería, Grupo de Investigación en tecnologías de las \\ Comunicaciones e Informática, GIMATICA, Avenida del Consulado, Calle 30, No 48 - 152, \\ Cartagena - Colombia.
}

Recibido Abr. 21, 2015; Aceptado Jun. 2, 2015; Versión final Ago. 28, 2015, Publicado Dic. 2015

\begin{abstract}
Resumen
El objetivo principal de este trabajo consiste en proponer una metodología para seleccionar aplicaciones VolP a implementar en pequeñas y medianas empresas. La metodología se aplicó al caso de una Pyme de agencia de viajes. Para esto se desarrollaron las siguientes fases: identificación de servicios ofrecidos, identificación de proveedores en el área geográfica, estudio de aplicaciones VoIP, estudio de procesos de comunicación de las Pymes y elaboración de la matriz para la selección de aplicaciones y proveedores. Los resultados de cada fase suministran datos necesarios para la ejecución de las siguientes etapas. Con el propósito de validar la utilidad y aplicación de la metodología propuesta, se llevaron a cabo pruebas para analizar la calidad de las aplicaciones VolP. Finalmente teniendo como base los resultados obtenidos, se encontró que la metodología propuesta es de gran utilidad para facilitar la selección de aplicaciones VolP, según la conveniencia de cada Pyme de acuerdo a sus procesos de comunicación y condiciones económicas.
\end{abstract}

\section{Methodology for Selecting Applications of the Communication Technology Voice over IP (VoIP) for Small and Medium Enterprises (SMEs). The Case of a Travel Agency}

\begin{abstract}
The aim of this paper is to propose a methodology for selecting VolP applications to be implemented in small and medium size enterprises. The methodology was applied to a representative SME such as a travel agency. To achieve this the following phases were developed: Identification of offered services, identification of providers in the geographic area, study VolP application, study communication processes of SMEs and development of the matrix for the selection of applications and providers. The results of each phase provide data necessary for the implementation of the following step. To validate the usefulness and applicability of the proposed methodology, tests were conducted to analyze the quality of VolP applications. Finally on the basis of the results obtained, it was found that the proposed methodology is useful to facilitate the selection of VolP applications, according to the convenience of each SME and of their communication processes and economic conditions.
\end{abstract}

Keywords: VolP technologies; communication processes; SMEs; quality of service 


\section{INTRODUCCIÓN}

Hoy día, las Tecnologías de comunicación tienen implicaciones a nivel empresarial (Jiménez, 2012). La comunicación en una organización es de vital importancia, debido a que fortalece el trabajo en equipo, contribuye a un mejor ambiente laboral y se logran mejores resultados dentro de las diferentes áreas (Hernandez-Ortega et al., 2014). En consecuencia, se tiene un aumento de productividad en las mismas, lo que se refleja en una organización fuerte, sólida y en crecimiento (Steinfield et al., 2010). El desarrollo de las TICs ha permitido el incremento de velocidad y fiabilidad de la información, dando paso a nuevas y mejores formas de comunicación, por tanto resulta frecuentemente adoptada la Tecnología VolP (Cecere et al., 2012). Esta tecnología es una categoría de hardware y software que permite usar internet como el medio de transmisión de llamadas telefónicas. La comunicación se logra enviando voz en paquetes de datos, usando el protocolo de Internet (IP), en lugar de la red conmutada (Goode, 2002).

La importancia del uso de las tecnologías VolP, para la comunicación por parte de las Pymes, radica en beneficios que pueden obtener con su implementación, como bajos costos y múltiples servicios (PBX, buzón de voz, entre otros) que le permiten sacar provecho al obtener mejores resultados (Dang et al, 2004). Sin embargo, y pese a los beneficios de VolP, muchas Pymes no la han adoptado, pero existen potenciales usuarios y otros que tienen conocimiento de ella (Ismail et al., 2011). En Cartagena Colombia, las Agencias de Viajes, desconocen esta tecnología. Teniendo en cuenta lo anterior, la importancia de esta investigación consiste en proponer una metodología para seleccionar aplicaciones VolP a implementar en dichas organizaciones. Lo anterior con la finalidad de determinar cuáles serían las opciones que mejor se adapten a sus procesos de comunicación y condiciones económicas. Además la metodología permite mostrar la utilidad y empleo de aplicaciones VolP, que cumplan con los requisitos funcionales de los procesos de comunicación encontrados en estas pymes, de manera que permita seleccionar la alternativa adecuada.

Como resultado de la metodología, se pudo verificar que las tecnologías VolP aportan múltiples beneficios como: disminución de costos por licencias, instalación y mantenimiento, mayor oferta de servicios, y utilización de la misma red para la transmisión de datos y voz (Dang et al., 2004); se logró la identificación de requisitos para la implementación de VoIP, gracias a la realización de una encuesta acerca de las TIC's utilizadas por las Pymes Agencias de Viajes en Cartagena, así como los servicios que ofrecen, y la manera en la que brindan los mismos. Adicionalmente se obtuvo una matriz de evaluación de las aplicaciones VolP, donde se muestran las características de las aplicaciones y las empresas proveedoras de servicio, lo que permite que cualquier Pyme interesada en implementar dichas tecnologías, pueda seleccionar de acuerdo a sus procesos de comunicación y condiciones económicas, la opción que considere favorable y disponible en el mercado.

\section{ESTUDIOS PATROCINADOS}

La VolP ha sido empleada en diversos campos a nivel mundial como solución de comunicación en organizaciones (Zhijun, 2015), se han realizado muchos trabajos y estudios alrededor de esta tecnología. En (Carrillo y López, 2011) se dimensiona una central telefónica VolP basada en Asterisk, que sería implementada a futuro por el Hospital Carlos Andrade Marín de la Ciudad de Quito-Ecuador, de acuerdo a un estudio previo sobre las necesidades reales del hospital para encontrar la mejor opción práctica y viable para una implementación inmediata. Por otro lado (Sinche et al., 2011) se realiza un análisis del rendimiento de sistemas VolP bajo condiciones de red variable para implementar una solución tecnológica, con el fin de monitorear una central telefónica Asterisk. En (castillo, 2011) se demuestra el funcionamiento y la aplicación de la telefonía IP en entornos corporativos, como es el caso de UNIBANCO S.A., mediante una solución Open Source. En (Kwak y Lee, 2011) se realiza un análisis empírico sobre la industria VolP en Corea, con la intención de proporcionar información valiosa para los países que buscan desarrollar su propia industria y las empresas que desarrollan nuevas estrategias basadas en el mercado de VolP. (Singh et al., 2014) Afirman que el sistema de Volp se ha establecido como la mejor alternativa para la comunicación, conclusión a la que llegaron luego de haber examinado los factores que afectan a la calidad de la señal Volp: condiciones de red, procesos de codificación, contenido de conversaciones y esquema de corrección de errores. En (Murazzo et al., 2013) también se aborda el tema de la calidad de los servicios en ambiente de redes VolP, evaluando el impacto de dos mecanismos de QoS (la inclusión de extensiones de QoS a protocolos existentes y la gestión de tráfico, que permite priorizar el tráfico proveniente de aplicaciones sensitivas) en ambientes de red cableadas e inalámbricas. Mediante un caso de estudio (García et al., 2014) realiza un análisis de la calidad de servicio (QoS) que perciben los usuarios Wimax cuando se interconectan a un Core MPLS. Los servicios de voz y video requieren de la asignación de recursos para transferir eficientemente la data, entre los que se encuentran la pérdida mínima de información y la reserva adecuada de ancho de banda. 
La integración de Redes Universitarias es un proyecto tecnológico llevado a cabo por Red Clara (Cooperación Latino Americana de redes avanzadas) en conjunto con RNP (Red Nacional de Educación e Investigación del Brasil), cuyo objetivo consiste en crear un punto de intercambio de tráfico telefónico entre las instituciones académicas latinoamericanas, empleando tecnologías VolP y Protocolos de comunicación abiertos y escalables que mejorarían la calidad de la comunicación y los costos (Martin y Aversa, 2013). Por otro lado también se han realizado trabajos relacionados con este artículo, (Salah, 2006) realizó un documento donde describe las directrices y una metodología paso a paso sobre cómo VolP puede ser desplegado con éxito. La metodología se puede utilizar para evaluar el apoyo y la disposición de una red existente. La metodología predice el número de llamadas VoIP que puede sostenerse por una red existente al tiempo que satisface los requisitos de calidad de todos los servicios de red y dejando una capacidad adecuada para el crecimiento futuro. (Ransome, 2005) habla sobre las etapas implicadas en la implementación de VolP, entre las que se encuentran la planificación como el primer paso necesario en cualquier proyecto de envergadura, la implementación y evaluación de aplicaciones teniendo en cuenta las necesidades que se quieren satisfacer, al igual que en (Walter J., 2002) explican lo que se debe tener en cuenta al planear una implementación de VolP y cómo realizar una correcta gestión. A pesar de la existencia de muchos trabajos similares, ninguno se ha enfocado en las Pymes Agencias de Viajes.

\section{PROPUESTA PLANTEADA}

En esta sección se presenta la metodología propuesta, para facilitar la selección de Tecnologías Voz sobre IP según los procesos de comunicación en las Pymes, situación que puede tornarse compleja al estar frente a múltiples aplicaciones VoIP que permiten varios servicios. El esquema de la metodología para la selección de aplicaciones VoIP se presenta en la figura 1. Como se puede apreciar la metodología propuesta consta de cinco fases, las cuales se detallan a continuación.

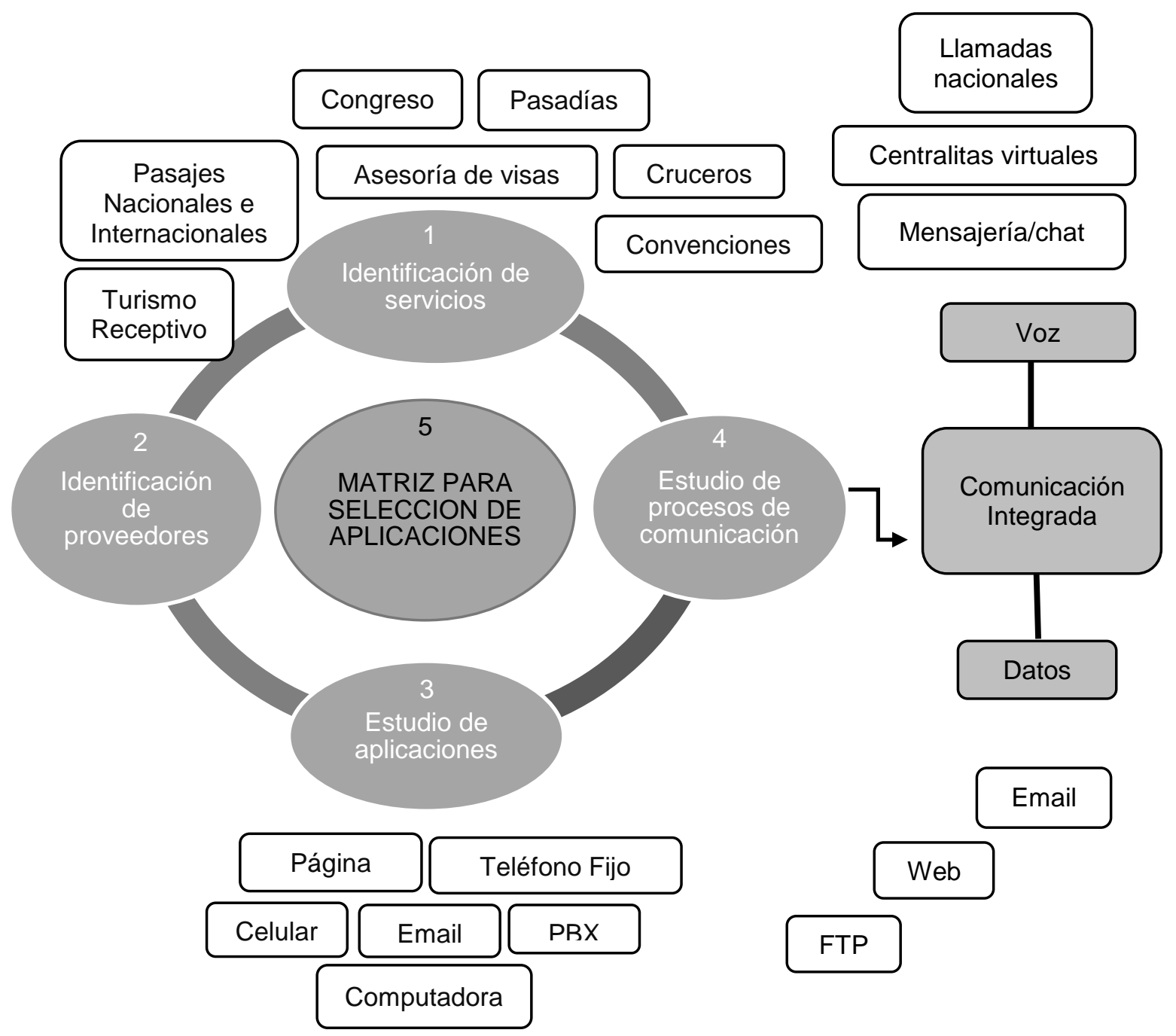

Fig. 1: Metodología Fuente: Autores de la investigación 
i) La primera fase consiste en identificar servicios ofrecidos por Pymes, para esto se realiza recolección de información a través de entrevistas a los administradores de ellas, con la cual se pretende determinar necesidades (servicios) en las Pymes y las aplicaciones Volp adecuadas para satisfacerlas.

ii) La segunda fase consiste en identificar cuáles son las empresas que brindan soporte a la implementación de Volp en el área geográfica en la cual se encuentra inmersa la Pyme en estudio, con el fin de buscar la mejor relación costo beneficio $(\mathrm{R} B / \mathrm{C})$. Para esto se recolecta información mediante entrevistas y consultas vía internet. Lo anterior es utilizado para identificar aspectos significativos como equipos de trabajo y costos, teniendo en cuenta las necesidades encontradas en la primera fase.

iii) La tercera fase consta de un estudio de aplicaciones VolP, con el fin de determinar las de mejor acople a las necesidades de las Pymes, para esto se realiza una recolección y análisis de información sobre aplicaciones desarrolladas alrededor del mundo para implementación de VolP.

iv) La cuarta fase consiste en identificar los procesos de comunicación de las Pymes para determinar en cuales procesos se justifica el uso de aplicaciones Volp, lo anterior es basado en un estudio de la relación costo beneficio del proceso a implementar.

v) La quinta fase consiste en elaborar una matriz que permite a cualquier tipo de empresa interesada en implementar esta tecnología VolP deducir que aplicaciones, procesos y proveedores seleccionar, teniendo en cuenta las necesidades encontradas y la relación beneficio-costo.

Los procesos de comunicación funcionales generados a partir del anterior modelo son los siguientes: Realizar llamadas nacionales e internacionales, Llamadas internas, enviar y recibir correo electrónico, y videoconferencias. Con el fin de atender los procesos funcionales expuestos, se realizó un análisis de aplicaciones de tecnología Voz sobre IP como son: 3CX, Xlite, y Zoiper; debido a que ellas permiten realizar servicios que ofrecen las organizaciones en estudio. También se incluyeron los proveedores que pueden dar soporte a la implementación de tecnologías VolP. Por tal razón, para garantizar que las aplicaciones seleccionadas prestan un mejor servicio, se realizaron pruebas para medir la calidad de la voz, bajo la normativa de la UIT-T, las recomendaciones UIT-T Y.1540 que define parámetros que permiten especificar y evaluar la calidad de funcionamiento tales como: retardo, Jitter, Pérdida de paquetes y Throughput; Y UIT-T Y.1541 que especifica los valores de calidad de funcionamiento de IP aceptables para cada uno de los parámetros de calidad de funcionamiento definidos en la Recomendación UIT-T Y.1540. Para determinar si cumple con calidad de la voz, las siguientes tablas representan los resultados de las aplicaciones evaluadas, el análisis consistió en realizar llamadas desde cada una de las aplicaciones seleccionadas, y obtener capturas mediante Wireshark. Cada llamada tuvo una duración aproximada de 2 minutos, el número de llamadas realizadas con cada aplicación fue de 10, cabe mencionar que fueron hechas en un entorno libre de ruidos provenientes de otras personas o maquinaria, un ancho de banda de 8MB, switch DGS 3100 48p, y Servidor HP ProLiant DL320e Gen8 v2 Server, se instalaron las aplicaciones en computadores de escritorio, celulares y Tabletas; para determinar si con el uso de: $3 \mathrm{CX}$, Xlite, y/o Zoiper, la calidad de la conversación no se interrumpe por fallas que se podrían presentar al no cumplir con parámetros que establece la norma mencionada. Al aplicar lo anteriormente descrito se obtuvo lo siguiente:

Tabla 1: Perdida de paquetes en aplicaciones VolP

\begin{tabular}{|c|c|c|}
\hline $\begin{array}{c}\text { Aplicacione } \\
s\end{array}$ & Máx. Paquetes perdidos (\%) & $\begin{array}{c}\text { Promedio de paquetes perdidos } \\
(\%)\end{array}$ \\
\hline 3CX & $0.004 \%$ & $0.0039 \%$ \\
\hline X-LITE & $0.0063 \%$ & $0.0037 \%$ \\
\hline ZOIPER & $0.0051 \%$ & $0.0038 \%$ \\
\hline
\end{tabular}

Tabla 2: Retardo en aplicaciones VolP

\begin{tabular}{|c|c|c|}
\hline Aplicaciones & Máx. Retardo $(\mathrm{ms})$ & Promedio de retardo $(\mathrm{ms})$ \\
\hline 3CX & 146.09 & 80.25 \\
\hline X-LITE & 147.21 & 81.03 \\
\hline ZOIPER & 146.12 & 81.48 \\
\hline
\end{tabular}


De acuerdo con la Tabla 1 las aplicaciones (3CX, X-lite y Zoiper) se obtuvo un promedio de perdida de paquetes $0.0039 \%, 0.0037 \%$ y $0.0038 \%$ respectivamente, siendo mínima la diferencia en la perdida de paquetes entre ellas, por lo anterior se puede inferir que al utilizar cualquiera de ellas no se presenta una degradación en la calidad de la conversación en una más que en otra. Además el máximo de paquetes perdidos de 3CX, X-LITE y ZOIPER están muy por debajo del promedio, para presentarse perdida en la calidad de la llamada como lo establece la clase 0 o clase 1 de la norma UIT-T Y.1541 que establece las recomendaciones para garantizar un óptimo servicio de VolP.

En relación con la tabla 2 los resultados de las tres aplicaciones (3CX, X-lite y Zoiper) se tiene que el máximo retardo encontrado en las aplicaciones que se realizó en las pruebas de Calidad de la voz fueron respectivamente: $80.25 \mathrm{~ms}, 81.03 \mathrm{~ms}$, y $81.48 \mathrm{~ms}$, dichos valores se encuentran dentro del rango aceptable para llamadas de baja interactividad como lo establece la UIT-T Y.1541 Clase 1, además el promedio de los retardos está por debajo de los $100 \mathrm{~ms}$. Teniendo una diferencia mínima en la calidad de las llamadas con cualquiera de ellas, como se puede apreciar en las columnas 2 y 3 de la Tabla 2. , lo que permite una fluidez en la llamada que se realiza. Con las aplicaciones $3 C X$, X-lite y Zoiper, se obtuvo un promedio de Jitter de $6.75 \mathrm{~ms}, 6.93 \mathrm{~ms}$, y $6.86 \mathrm{~ms}$ respectivamente con el tráfico de red saturado, no se percibió degradación en el servicio de VolP. Cabe destacar que tras las pruebas, $3 \mathrm{CX}$ presento mejores resultados que X-LITE y ZOIPER aplicaciones con las que fue comparada.

\section{RESULTADOS}

Con el fin de dar cumplimiento al objetivo principal de este artículo, se desarrolló una matriz de evaluación de aplicaciones VOIP. Esta matriz muestra a las Pymes, una lista de aplicaciones que cumplen y se ajustan a los procesos de comunicación, encontrados en estas empresas. Además, de los proveedores que a nivel nacional prestan el servicio de soporte a estas tecnologías. Lo que permite que tengan diversas opciones funcionales, entre las cuales elegir la que consideren más conveniente para su empresa, según sus recursos económicos, los servicios que brinden y funcionalidades que requieran.

La Tabla 3, muestra la matriz elaborada y las características que permiten una toma de decisiones por parte de las empresas. En esta matriz se visualiza dos tipos de aplicaciones VoIP, que pueden ser de tipo servidor o tipo Cliente, en la cual se detallan las características que estas tienen y que permiten prestar los servicios que se dispone en las Pymes agencias de viajes. Se proporciona información de estas aplicaciones para un mejor análisis al momento de elegir, como: el idioma, licencia, protocolos que emplea, grado de calidad, entre otros. Además se muestra los proveedores que a nivel nacional prestan soporte técnico a servicios VolP, de esta manera se le facilita a las empresas: conocer, contactar y solicitar sus servicios al decidir implementar una o algunas de las tecnologías VolP presentadas en la Tabla 3.

Para mostrar el uso de la matriz se tomó una de las agencias entrevistada: Costa Tur Ltda. Una compañía con 10 años de experiencia en el mercado turístico. Enfocados especialmente al manejo de turismo receptivo, congresos y convenciones, e incentivos; servicios en turismo receptivo incluyen: Reservas Aéreas, Reservas Hoteleras, Reservas en Restaurantes, Excursiones Opcionales, Paquetes Turísticos, Traslados, Alquiler de Vehículos. Para la comunicación con los clientes, proveedores y entre empleados de la empresa, utilizan e-mail, página web, llamadas telefónicas y PBX.

Dada la forma en que contactan a sus clientes y proveedores el uso de softphone como LINPHONE, XLITE, 3CXPHONE y ZOIPER, les permite realizar llamadas nacionales e internacionales, mensajería instantánea, instalación en móvil, video llamadas y licencia GPL. Además pueden ser ejecutados en distintos sistemas operativos a excepción de 3CXPHONE, que permite solo Windows y móvil, pero cuenta con la característica de servir como extensión de una PBX; además de ser compatibles con el protocolo SIP, lo que facilita la configuración con la central telefónica que podría ser ASTERISK o 3CX. Las dos anteriores tienen buenos niveles de calidad, compatibilidad con diversos protocolos, pero deben tener en cuenta que algunas funciones o servicios de 3CX son comerciales.

Además de los servicios que ofrece esta agencia, los proveedores que a nivel nacional dan soporte a esos servicios VolP son: Movilcom Colombia, Voz Ip Store S.A.S, Voipmundo Ltda., Linkedip Ltda., como se muestra en la Tabla 4, en la que se visualiza que servicios ofrece cada proveedor. Tener información de antemano de las empresas que prestan Soporte a tecnologías VolP, será útil al momento de querer implementar estas tecnologías y requerir de sus servicios. 
Tabla 3: Matriz de Evaluación de Aplicaciones VOIP

\begin{tabular}{|c|c|c|c|c|c|c|c|c|c|c|}
\hline \multirow[t]{2}{*}{ Nombre } & \multicolumn{2}{|c|}{ Aplicación servidor } & \multicolumn{7}{|c|}{ Aplicación cliente } & \multirow[t]{2}{*}{$\begin{array}{c}\text { Proveedore } \\
\mathrm{S}\end{array}$} \\
\hline & $\begin{array}{c}\text { ASTERI } \\
\text { SK }\end{array}$ & $\begin{array}{l}\text { CENTRAL } \\
\text { TELEFON } \\
\text { ICA 3CX }\end{array}$ & JITSI & X-LITE & $\begin{array}{l}\text { QUTEC } \\
\text { OM }\end{array}$ & $\begin{array}{c}\text { 3XC } \\
\text { PHONE }\end{array}$ & $\begin{array}{c}\text { LINPHO } \\
\text { NE }\end{array}$ & ZOIPER & FRING & \\
\hline $\begin{array}{l}\text { Llamadas } \\
\text { nacionales } \\
\text { e } \\
\text { internacion } \\
\text { ales } \\
\end{array}$ & $\mathrm{Si}$ & Si & Si & $\mathrm{Si}$ & $\mathrm{Si}$ & $\mathrm{Si}$ & $\mathrm{Si}$ & $\mathrm{Si}$ & $\mathrm{Si}$ & \multirow{13}{*}{$\begin{array}{c}\text { Linkedip } \\
\text { Ltad. } \\
\text { (Cartagena) } \\
\text { y } \\
\text { Voipmundo } \\
\text { Ltda. } \\
\text { (Bogotá) } \\
\text { Instalación } \\
\text { y soporte } \\
\text { de } \\
\text { Aplicacione } \\
\text { s servidor y } \\
\text { Cliente. } \\
\\
\text { E-mail } \\
\text { Corporativo } \\
\text { integrado a } \\
\text { la } \\
\text { plataforma } \\
\text { VolP } \\
\text { mediante } \\
\text { servidor de } \\
\text { correo. } \\
\text { (Postfix) } \\
\text { Llamadas } \\
\text { nacionales } \\
\text { e } \\
\text { internacion } \\
\text { ales. }\end{array}$} \\
\hline $\begin{array}{c}\text { Correo } \\
\text { electronico }\end{array}$ & Si & $\mathrm{Si}$ & No & No & No & No & No & No & No & \\
\hline Video & $\mathrm{Si}$ & Si & $\mathrm{Si}$ & $\mathrm{Si}$ & Si & $\mathrm{Si}$ & Si & $\begin{array}{l}\text { No - } \\
\text { Si(comer } \\
\text { cial) }\end{array}$ & $\mathrm{Si}$ & \\
\hline $\begin{array}{c}\text { Grado de } \\
\text { calidad }\end{array}$ & Bueno & Bueno & Bueno & Bueno & Regular & Bueno & Regular & Bueno & Bueno & \\
\hline Licencia & Free & $\begin{array}{c}\text { Free y } \\
\text { Comercia } \\
\text { I }\end{array}$ & Free & $\begin{array}{c}\text { Free y } \\
\text { Comerc } \\
\text { ial }\end{array}$ & Free & $\begin{array}{c}\text { Free y } \\
\text { Comer } \\
\text { cial }\end{array}$ & Free & $\begin{array}{c}\text { Free y } \\
\text { Comercia } \\
1\end{array}$ & $\begin{array}{l}\text { Comer } \\
\text { cial }\end{array}$ & \\
\hline Escritorio & $\begin{array}{c}\text { Linux. } \\
\text { BSD, } \\
\text { Mac } \\
\text { OS X, } \\
\text { Solaris } \\
\text { y } \\
\text { Windo } \\
\text { ws }\end{array}$ & Windows & $\begin{array}{l}\text { Linux, } \\
\text { Mac y } \\
\text { Windo } \\
\text { ws }\end{array}$ & $\begin{array}{l}\text { Linux, } \\
\text { Mac y } \\
\text { Window } \\
\text { s }\end{array}$ & $\begin{array}{l}\text { Linux, } \\
\text { Mac y } \\
\text { Windo } \\
\text { ws }\end{array}$ & $\begin{array}{l}\text { Windo } \\
\text { ws }\end{array}$ & $\begin{array}{l}\text { Linux, } \\
\text { Mac y } \\
\text { Window } \\
\text { s }\end{array}$ & $\begin{array}{c}\text { Linux, } \\
\text { Mac y } \\
\text { Windows }\end{array}$ & No & \\
\hline Movil & NO & NO & No & $\begin{array}{c}\text { Android } \\
\text {, iOS y } \\
\text { Blackbe } \\
\text { rry }\end{array}$ & No & $\begin{array}{c}\text { Android } \\
\text {, iOS }\end{array}$ & $\begin{array}{c}\text { Android } \\
\text {, iOS y } \\
\text { Blackbe } \\
\text { rry }\end{array}$ & $\begin{array}{l}\text { Android, } \\
\text { iOS }\end{array}$ & $\begin{array}{c}\text { Androi, } \\
\text { iOS y } \\
\text { Symbia } \\
n\end{array}$ & \\
\hline Protocolos & $\begin{array}{l}\text { SIP, } \\
\text { H.323, } \\
\text { IAXY } \\
\text { MGCP }\end{array}$ & SIP & $\begin{array}{l}\text { SIP, } \\
\text { XMPP }\end{array}$ & $\begin{array}{l}\text { SIP, } \\
\text { IAX }\end{array}$ & $\begin{array}{l}\text { SIP, } \\
\text { XMPP }\end{array}$ & SIP & $\begin{array}{l}\text { SIP, } \\
\text { XMPP }\end{array}$ & $\begin{array}{c}\text { IAX, SIP, } \\
\text { XMPP } \\
\text { (comercia } \\
\text { I) }\end{array}$ & $\begin{array}{c}\text { SIP, } \\
\text { XMPP }\end{array}$ & \\
\hline Mensajeria & $\mathrm{Si}$ & $\mathrm{Si}$ & $\mathrm{Si}$ & $\mathrm{Si}$ & Si & $\mathrm{Si}$ & $\mathrm{Si}$ & $\mathrm{Si}$ & $\mathrm{Si}$ & \\
\hline $\begin{array}{c}\text { Conferenci } \\
\text { a }\end{array}$ & $\mathrm{Si}$ & Si & $\mathrm{Si}$ & Si & Si & Si & No & Si & $\mathrm{Si}$ & \\
\hline Idioma & Ingles & $\begin{array}{l}\text { Inglés y } \\
\text { Español }\end{array}$ & $\begin{array}{c}\text { Españ } \\
\text { ol }\end{array}$ & Ingles & $\begin{array}{c}\text { Españo } \\
\text { I }\end{array}$ & Multi. & Ingles & Ingles & Multi. & \\
\hline Extension & & & No & $\mathrm{Si}$ & No & $\mathrm{Si}$ & No & $\mathrm{Si}$ & No & \\
\hline PBX & SI & SI & No & No & No & No & No & No & No & \\
\hline
\end{tabular}

Tabla 4: Proveedores de Servicios VolP

\begin{tabular}{|c|c|c|c|c|}
\hline NOMBRE & $\begin{array}{c}\text { MOVILCOM } \\
\text { COLOMBIA }\end{array}$ & $\begin{array}{c}\text { VOZ IP STORE } \\
\text { S.A.S }\end{array}$ & $\begin{array}{c}\text { VOIPMUNDO } \\
\text { LTDA. }\end{array}$ & $\begin{array}{c}\text { LINKEDIP } \\
\text { LTDA. }\end{array}$ \\
\hline $\begin{array}{c}\text { Llamadas nacionales e } \\
\text { internacionales }\end{array}$ & $\mathrm{X}$ & $\mathrm{X}$ & $\mathrm{X}$ & $\mathrm{X}$ \\
\hline Email corporativo & & $\mathrm{X}$ & $\mathrm{X}$ & $\mathrm{X}$ \\
\hline $\begin{array}{c}\text { Instalación y soporte de } \\
\text { aplicaciones voip servidor }\end{array}$ & & $\mathrm{X}$ & $\mathrm{X}$ & $\mathrm{X}$ \\
\hline $\begin{array}{c}\text { Instalación y soporte de } \\
\text { aplicaciones voip cliente }\end{array}$ & & $\mathrm{X}$ & $\mathrm{X}$ \\
\hline Número entrante & & & $\mathrm{X}$ \\
\hline $\begin{array}{c}\text { Localizadas en la ciudad de } \\
\text { cartagena }\end{array}$ & & & \\
\hline
\end{tabular}




\section{CONCLUSIONES}

1. Se pudo comprobar la utilidad que presenta la metodología propuesta como una herramienta a las Pymes Agencias de Viaje en la selección de aplicaciones Volp para la implementación de Servicios. 2. Con la determinación de las fases de la metodología propuesta se esquematiza para ser utilizada en cualquier tipo de Pyme, solo es tener en cuenta la aplicación de la misma. 3. Al utilizar la metodología se denota la importancia que presenta la Tecnología Volp en los procesos de comunicación de la Pyme. 4. La importancia de realizar pruebas a las aplicaciones Volp en el entorno que se van a implementar los servicios, es para analizar la calidad del mismo y con ello obtener una eficaz elección de las aplicaciones de acuerdo a: necesidades, entorno y condiciones económicas.

\section{REFERENCIAS}

Carrillo Armendáriz, E. P., y López Hernández, W. G., Análisis, rediseño de la red Lan y dimensionamiento de una central telefónica de Voip basado en software libre (Asterisk) para el Hospital Carlos Andrade Marín de la ciudad de Quito, Fac. Ing. Eléctrica y Electrónica, Escuela Politécnica Nacional, Ecuador (2011)

Castillo Caiza, J. F., Estudio, análisis y diseño de una solución basada en tecnología de VOIP para la agencia Ejido del Banco Universal SA, y su integración con las principales agencias, Tesis de grado, Dpto. Ing. Sistemas, Universidad Politécnica Salesiana, Ecuador (2011)

Cecere, G., y Corrocher, N., The usage of VolP services and other communication services: An empirical analysis of Italian consumers, Technological Forecasting and Social Change, 79(3), 570-578 (2012)

Dang, T. D., Sonkoly, B., y Molnár, S., Fractal analysis and modeling of VolP traffic, In Telecommunications Network Strategy and Planning Symposium, NETWORKS 2004, 11th International (pp. 123-130), IEEE, June (2004)

García, R. C., Salcedo, O. J., López, D. A., y Pedraza, L. F., Evaluación de la Calidad del Servicio para Voz sobre Protocolo de Internet (VoIP) en Redes WIMAX sobre Ambientes IP/MPLS, doi: 10.4067/S071807642014000200004, Información tecnológica, 25(2), 24-38 (2014)

Goode, B., Voice over internet protocol (VoIP), Proceedings of the IEEE, 90(9), 1495-1517 (2002)

Hernandez-Ortega, B., Serrano-Cinca, C., y Gomez-Meneses, F., The firm's continuance intentions to use inter-organizational ICTs: The influence of contingency factors and perceptions, Information \& Management, 51(6), 747-761 (2014)

Ismail, R., Jeffery, R., y Van Belle, J., Using ICT as a value adding tool in South African SMEs, Journal of African Research in Business \& Technology, 1-11 (2011)

Jiménez, I. E. L., El Impacto de la Tecnología en la Comunicación Empresarial: Reflexiones y Análisis, Razón y Palabra, 17(79) (2012)

Kwak, J. H., y Lee, B. G., Estimating demand curve in the Korean VolP telecommunications market, Technological Forecasting and Social Change, 78(4), 713-728 (2011)

Martín, M. J., y Aversa, F., Redes académicas de VolP latinoamericanas frente al desafío de las nuevas tecnologías, Tercera Conferencia de Directores de Tecnología de Información, TICAL 2013 Gestión de las TICs para la Investigación y la Colaboración, Cartagena de Indias, Colombia 8 y 9 de Julio (2013)

Murazzo, M. A. y otros 6 autores, Administración de QoS en ambientes de redes de servicios convergentes, In XV Workshop de Investigadores en Ciencias de la Computación, Junio (2013)

Ransome, J. F., CISM, C., y Rittinghouse, J., Voice over Internet Protocol (VoIP) Security, Digital Press, (2005)

Recommendation ITU-T Y.1540, Internet protocol data communication service -IP packet transfer and availability performance parameters, 16-27, Ginebra, Suiza (2011)

Recommendation ITU-T Y.1541, Network performance objectives for IP-based Services, 34-35, Ginebra, Suiza (2011) 
Salah, K., On the deployment of VolP in Ethernet networks: methodology and case study, doi:10.1016/j.comcom.2005.06.004, Computer Communications, 29(8), 1039-1054 (2006)

Sinche, E., Ortega, J., y Astudillo, M. G., Análisis del rendimiento de sistemas voip bajo condiciones de red variable, Tesis de grado, Escuela superior Politécnica del Litoral, Fac. de Ingeniería en Electricidad y Computación (2011)

Singh, H. P., Singh, S., Singh, J., y Khan, S. A., VolP: State of art for global connectivity-A critical review, doi:10.1016/j.jnca.2013.02.026, Journal of Network and Computer Applications, 37, 365-379 (2014)

Steinfield, C., Scupola, A., y López-Nicolás, C., Social capital, ICT use and company performance: Findings from the Medicon Valley Biotech Cluster, Technological Forecasting and Social Change, 77(7), 1156-1166 (2010)

Walker, J. Q., y Hicks, J. T., The Essential Guide to VolP implementation and management, NetlQ Corporation (2002)

Zhijun, w., Covert Communication Based on the VolP System, doi:10.1016/B978-0-12-801328-1.00008-2, Information Hiding in Speech Signal for Secure Communication, 127-142 (2015) 